\title{
EMT: When epithelial cells decide to become mesenchymal-like cells
}

\author{
Raghu Kalluri1,2
}

\begin{abstract}
${ }^{1}$ Division of Matrix Biology, Beth Israel Deaconess Medical Center, and Department of Biological Chemistry and Molecular Pharmacology, Harvard Medical School, Boston, Massachusetts, USA. ${ }^{2}$ Harvard-MIT Division of Health Sciences and Technology, Boston, Massachusetts, USA.
\end{abstract}

\begin{abstract}
Epithelial-mesenchymal transition (EMT) is critical for appropriate embryonic development, and this process is re-engaged in adults during wound healing, tissue regeneration, organ fibrosis, and cancer progression. Inflammation is a crucial conspirator in the emergence of EMT in adults but is absent during embryonic development. As highlighted in this Review series, EMT is now a recognized mechanism for dispersing cells in embryos, forming fibroblasts/mesenchymal cells in injured tissues, and initiating metastasis of epithelial cancer cells. Also discussed are proposals to classify EMT into three subtypes, each of which has different functional consequences.
\end{abstract}

Mesenchymal cells are unique spindle-shaped cells that exhibit end-to-end polarity. Fibroblasts are prototypical mesenchymal cells that exist in many tissues and get activated during repair processes (1). Fibroblasts/mesenchymal cells were the first cell type(s) to be cultured on glass and plastic and were subsequently used in many studies to evaluate intracellular signaling, cytoskeletal organization, and cell-cell and cell-ECM interactions. Nevertheless, fibroblasts/mesenchymal cells still represent one of the least understood types of cell(s) at the molecular level, and protein markers specific for these cells are still lacking (1). Moreover, the origin of fibroblasts/mesenchymal cells during development, tissue repair, and pathological insults is not completely understood. As discussed in this Review series, epithelial-mesenchymal transition (EMT) is one mechanism by which cells with mesenchymal features can be generated in numerous different settings.

\section{The need for EMT}

All cells in our body are derived from a single cell, and phenotypic variants are a result of specific expression of a defined transcriptome that facilitates further functional diversity. During embryogenesis, epithelia are considered to be highly plastic and able to switch back and forth between epithelia and mesenchyme, via the processes of EMT and mesenchymal-epithelial transition (MET), respectively $(2,3)$. Upon completion of organ development epithelia typically serve specialized functions $(4,5)$. For decades it has been proposed that a state of terminal differentiation is necessary for epithelia to execute these functions and that this state is protected once it has been reached. This formidable notion was based on conjecture without any experimental evidence and has been challenged by observations that terminally differentiated epithelia can change their phenotype under the influence of repair-associated or pathological stress (6-8). One phenomenon that facilitates such cellular diversity during development and adulthood is EMT, the basics of which are discussed by Robert Weinberg and myself in the first article in this Review series (9). Thus, EMT is now a recognized mechanism for dispersing cells in

Conflict of interest: The author has declared that no conflict of interest exists.

Nonstandard abbreviations used: EMT, epithelial-mesenchymal transition; MET, mesenchymal-epithelial transition.

Citation for this article: J. Clin. Invest. 119:1417-1419 (2009). doi:10.1172/JCI39675. embryos, forming fibroblasts/mesenchymal cells in injured tissues, and initiating invasive and metastatic behavior in epithelial cancer cells (10-13). The process of EMT, which generates morphologically and functionally distinct cell types during embryogenesis, is inextricably linked to the evolutionary pressures that led to the emergence of metazoans. In the adult, it occurs during wound healing, tissue regeneration, and organ fibrosis to generate repair-associated mesenchymal cells/fibroblasts. Changes in the genome of an epithelial cancer cell place the cell under pressure to move locally and systemically, and this process is facilitated by EMTs that generate cancer cell variants.

The biological pressure for EMT is therefore to generate cells with a distinct phenotype and tissue function, to facilitate movement of cells, to invade local tissue, and to enable genetically unstable cancer cells to acquire a mesenchymal phenotype to facilitate local and systemic dissemination. Interestingly, EMT during embryogenesis occurs in an immunologically privileged setting driven by internal molecular programs. Immune privilege might be necessary for EMT associated with embryo development, whereas inflammation and epigenetics are likely key inducers of EMT in the pathological settings of organ fibrosis and cancer progression.

\section{Typing EMTs}

Although the specific signals that delineate EMTs in discrete settings have not been yet been defined, it is clear that EMTs are encountered in three distinct settings with different functional consequences (Figure 1). A proposal to classify EMTs into three subtypes based on the biological and biomarker context in which they occur was discussed at a 2007 meeting on EMT in Poland and at a subsequent conference in March 2008 at Cold Spring Harbor Laboratories. EMTs associated with implantation, embryo formation, and organ development occur during a self-contained process with an implicit need to generate diverse cell types that share mesenchymal phenotypes and biomarkers. As proposed in this Review series, by Weinberg and myself (9) and Michael Zeisberg and Eric Neilson (14), such EMTs constitute one EMT subtype termed type 1 EMT. Type 1 EMT causes neither fibrosis nor uncontrolled systemic invasion by high-grade epithelial cancer cells; rather, it generates cells with a mesenchymal phenotype to create new tissue(s) with diverse functions. In this regard, the generation of melanocytes from neural crest cells via EMT results in an invasive 


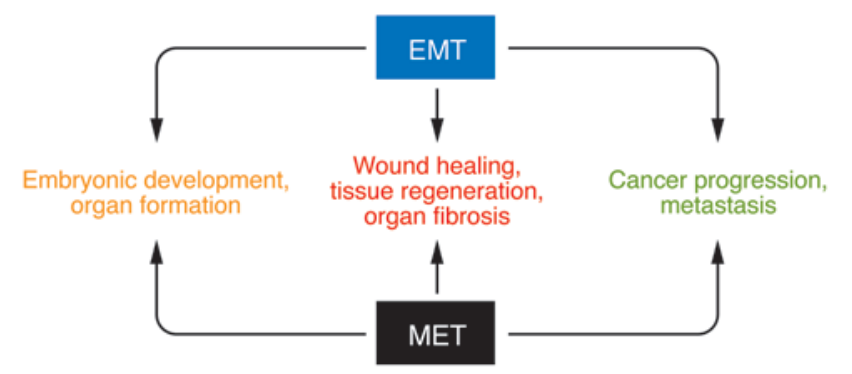

Figure 1

EMT and MET in health and disease. The evidence for EMT is compelling in embryonic development, organ formation, wound healing, tissue regeneration, organ fibrosis, cancer progression, and metastasis. The role for MET in wound healing, tissue regeneration, organ fibrosis, cancer progression, and metastasis is only speculated and rigorous evidence is still lacking.

phenotype with an important distinction from that of the metastatic cells that result from EMT involving primary epithelial cancer cells. Melanocytes do not invade via the circulation as epithelial cancer cells do. During development, EMT plays a critical role in generating the first set of mesenchymal cells, which are known as the primary mesenchyme. Subsequently, as tissue expands and specifications emerge, primary mesenchyme gives rise to secondary epithelia via MET, as is encountered during kidney development (15). In the final article in this Review series, Marianne BronnerFraser, M. Angela Nieto, and colleagues discuss the importance of EMT in development and highlight the signaling circuitry involved in EMTs in this context (16). These authors also suggest that the occurrence of EMT in adults is likely to be a reactivation of developmental paradigms, albeit with fatal consequences.

EMTs associated with wound healing, tissue regeneration, and organ fibrosis are proposed to constitute the second EMT subtype, type 2 EMT $(9,14)$. As discussed in this Review series by Weinberg and myself (9), EMTs in this context begin as part of a repair-associated event to generate fibroblasts to reconstruct and repair tissue following trauma and/or inflammatory injury. Type 2 EMT associated with wound healing, and presumably tissue regeneration, is associated with inflammation and ceases once repair is achieved and inflammation is attenuated. In the setting of organ fibrosis, type 2 EMT can continue to respond to ongoing inflammation, leading eventually to organ destruction.

Type 3 EMT is the third proposed subtype of EMT $(9,14)$, and it occurs in epithelial cancer cells that differ genetically and epigenetically from untransformed epithelial cells. As discussed in this Review series by Weinberg and myself (9), these changes, which mainly affect oncogenes and tumor suppressor genes, conspire with the EMT regulatory circuitry to produce outcomes far different from those observed in the other two types of EMT. Cells generated by type 3 EMT may invade and metastasize via the circulation and thereby generate systemic manifestations of malignant cancer progression. Although much is known about the signaling pathways involved in type 1 and type 2 EMT (9, $16)$, it is still unclear what specific signals induce type 3 EMT in epithelial carcinoma cells.

While these three classes of EMTs represent distinct biological processes, a common set of genetic and biochemical elements appears to underlie, and thus enable, these outwardly diverse phenotypic programs $(9,14,16)$. An in-depth analysis of what is known about biomarkers of the three types of EMTs, both those that are common among the different types of EMT and those that are unique to one type of EMT, is provided in this Review series by Zeisberg and Neilson (14). However, as experiments are performed in the future, we are sure to learn more regarding the similarities and the dissimilarities associated with the three classes of EMTs.

\section{Moving forward: EMTs in vivo}

As is clear from the articles in this Review series, the evidence for EMTs is overwhelming in experiments conducted in cell culture and rodents. However, pathologists still debate the relevance of EMT to human conditions such as organ fibrosis and cancer progression. This is because of difficulties in identifying transitioning epithelial cells passing through the basement membrane during organ fibrosis and detecting local or migratory cancer cells with a mesenchymal phenotype. Recent human studies using powerful imaging techniques and reliable cell-labeling methods have begun to unravel the occurrence of EMTs in organ fibrosis and cancer metastasis (17-21). Once the migratory cancer cells generated by type 3 EMT find themselves in distant tissue beds, they form secondary tumors exhibiting an epithelial phenotype. This suggests that the reversibility of EMT observed during embryonic development, when migratory mesenchyme gives rise to secondary epithelia, is also operational in the formation of secondary metastatic nodule. Such MET is poorly understood in the context of metastasis. In this regard, EMT/MET research in the coming years promises to be exciting, as new mouse models, molecular probes, and human studies are designed to address unanswered questions.

Inherent to an understanding of the contribution of EMT to human health and disease is the pressing need to appreciate the precise molecular and functional features of the emerging mesenchymal cells. Such need is tied to many fundamental questions that remain unanswered. What is the molecular definition of a mesenchymal cell resulting from EMT and that of a fibroblast? What are the molecular differences between mesenchymal cells generated by EMT and fibroblasts? Is a fibroblast a fully differentiated mesenchymal cell? What is the origin of fibroblasts? Do they come from immune cells? Are there links between mesenchymal cells and hematopoietic cells? Do fibroblasts derive from a stem cell shared by all mesenchymal cells? Answers will likely emerge in the near future.

\section{Acknowledgments}

The research work in the Kalluri laboratory is supported by the NIH grants DK55001, DK62978, DK61688, AA13913, and CA125550 and by research funds of the Division of Matrix Biology at the Beth Israel Deaconess Medical Center.

Address correspondence to: Raghu Kalluri, Harvard Medical School, Division of Matrix Biology, Beth Israel Deaconess Medical Center, 3 Blackfan Circle, CLS 11801, Boston, Massachusetts 02215, USA. Phone: (617) 667-0445; Fax: (617) 975-5663; E-mail: rkalluri@bidmc.harvard.edu.

1. Kalluri, R., and Zeisberg, M. 2006. Fibroblasts in cancer. Nat. Rev. Cancer. 6:392-401.

2. Thiery, J.P. 2002. Epithelial-mesenchymal transitions in tumour progression. Nat. Rev. Cancer. 2:442-454. 
3. Kalluri, R., and Neilson, E.G. 2003. Epithelialmesenchymal transition and its implications for fibrosis. J. Clin. Invest. 112:1776-1784.

4. Gumbiner, B.M. 1992. Epithelial morphogenesis. Cell. 69:385-387.

5. Yeaman, C., Grindstaff, K.K., Hansen, M.D., and Nelson, W.J. 1999. Cell polarity: Versatile scaffolds keep things in place. Curr. Biol. 9:R515-R517.

6. Boyer, B., Valles, A.M., and Edme, N. 2000. Induction and regulation of epithelial-mesenchymal transitions. Biochem. Pharmacol. 60:1091-1099.

7. Nieto, M.A. 2002. The snail superfamily of zincfinger transcription factors. Nat. Rev. Mol. Cell Biol. 3:155-166.

8. Tsai, R.Y., Kittappa, R., and McKay, R.D. 2002. Plasticity, niches, and the use of stem cells. Dev. Cell. 2:707-712.

9. Kalluri, R., and Weinberg, R.A. 2009. The basics of epithelial-mesenchymal transition. J. Clin. Invest. 119:1420-1428.

10. Kiemer, A.K., Takeuchi, K., and Quinlan, M.P. 2001. Identification of genes involved in epithelial- mesenchymal transition and tumor progression. Oncogene. 20:6679-6688.

11. Janda, E., et al. 2002. Ras and TGF[beta] cooperatively regulate epithelial cell plasticity and metastasis: dissection of Ras signaling pathways. J. Cell Biol. 156:299-313.

12. Vincent-Salomon, A., and Thiery, J.P. 2003. Host microenvironment in breast cancer development: epithelial-mesenchymal transition in breast cancer development. Breast Cancer Res. 5:101-106.

13. Xue, C., Plieth, D., Venkov, C., Xu, C., and Neilson, E.G. 2003. The gatekeeper effect of epithelial-msenchymal transition regulates the frequency of breast cancer metastases. Cancer Res. 63:3386-3394.

14. Zeisberg, M., and Neilson, E.G. 2009. Biomarkers for epithelial-mesenchymal transitions. J. Clin. Invest. 119:1429-1437.

15. Ekblom, P. 1996. Genetics of kidney development. Curr. Opin. Nephrol. Hypertens. 5:282-287.

16. Acloque, H., Adams, M.S., Fishwick, K., Bronner-Fraser, M., and Nieto, M.A. 2009. Epithelial-mesenchymal transitions: the importance of changing cell state in development and disease. J. Clin. Invest. 119:1438-1449.

17. Rastaldi, M.P., et al. 2002. Epithelial-mesenchymal transition of tubular epithelial cells in human renal biopsies. Kidney Int. 62:137-146.

18. Bataille, F., et al. 2008. Evidence for a role of epithelial mesenchymal transition during pathogenesis of fistulae in Crohn's disease. Inflamm. Bowel Dis. 14:1514-1527.

19. Reckamp, K.L., et al. 2008. Tumor response to combination celecoxib and erlotinib therapy in non-small cell lung cancer is associated with a low baseline matrix metalloproteinase- 9 and a decline in serum-soluble E-cadherin. J. Thorac. Oncol. 3:117-124.

20. Brabletz, T., et al. 2001. Variable beta-catenin expression in colorectal cancers indicates tumor progression driven by the tumor environment. Proc. Natl. Acad. Sci. U. S. A. 98:10356-10361.

21. Trimboli, A.J., et al. 2008. Direct evidence for epithelial-mesenchymal transitions in breast cancer. Cancer Res. 68:937-945. 